\title{
Evaluation of an Experimentally Designed Stereotactic Guidance System for Determining Needle Entry Point during Uniplanar Fluoroscopy-guided Intervention
}

\author{
Departments of Biomedical Engineering, *Anesthesia and Pain Medicine, \\ School of Medicine, Pusan National University, Yangsan, Korea
}

Jae Heon Lee, Gye Rok Jeon, Jung Hoon Ro, Gyeong Jo Byoen, MD*, Tae Kyun Kim, MD*, and Kyung Hoon Kim, MD*

\section{Background:}

In discography performed during percutaneous endoscopic lumbar discectomy (PELD) via the posterolateral approach, it is difficult to create a fluoroscopic tunnel view because a long needle is required for discography and the guide-wire used for consecutive PELD interrupts rotation of fluoroscope. A stereotactic system was designed to facilitate the determination of the needle entry point, and the feasibility of this system was evaluated during interventional spine procedures.

\section{Methods:}

A newly designed stereotactic guidance system underwent a field test application for PELD. Sixty patients who underwent single-level PELD at L4-L5 were randomly divided into conventional or stereotactic groups. PELD was performed via the posterolateral approach using the entry point on the skin determined by premeasured distance from the midline and angles according to preoperative magnetic resonance imaging (MRI) findings. Needle entry accuracy provided by the two groups was determined by comparing the distance and angle measured by postoperative computed tomography with those measured by preoperative MRI. The duration and radiation exposure for determining the entry point were measured in the groups.

Results:

The new stereotactic guidance system and the conventional method provided similarly accurate entry points for discography and consecutive PELD. However, the new stereotactic guidance system lowered the duration and radiation exposure for determining the entry point.

\section{Conclusions:}

The new stereotactic guidance system under fluoroscopy provided a reliable needle entry point for discography and consecutive PELD. Furthermore, it reduced the duration and radiation exposure associated with determining needle entry. (Korean J Pain 2012; 25: 81-88)

\section{Key Words:}

equipment design, fluoroscopy, needle, percutaneous discectomy, stereotactic technique.

Received January 9, 2012. Revised March 11, 2012. Accepted March 13, 2012.

Correspondence to: Kyung Hoon Kim, MD

Pain Clinic, Pusan National University Yangsan Hospital, Bumeu-ri, Mulgeum-eup, Yangsan 626-770, Korea

Tel: +82-55-360-2753, Fax: +82-55-360-2149, E-mail: pain@pusan.ac.kr

() This is an open-access article distributed under the terms of the Creative Commons Attribution Non-Commercial License (http:// creativecommons.org/licenses/by-nc/3.0/), which permits unrestricted non-commercial use, distribution, and reproduction in any medium, provided the original work is properly cited.

Copyright (c) The Korean Pain Society, 2012 


\section{INTRODUCTION}

Physicians are accustomed to fluoroscopic-guided intervention using the "bull's-eye" or tunnel-view technique, a coaxial technique in which a needle is parallel to the X-ray beam. However, it is difficult to create a fluoroscopic tunnel view for a 6 -inch long, 18-gauge needle in cases of discography performed during percutaneous endoscopic lumbar discectomy (PELD).

A full-rotation, 3-dimensional intraoperative image (O-arm) system with the capacity of combining 2-dimensional fluoroscopy imaging and 3-dimensional computed tomography (CT) imaging has recently become available [1]. However, there is little space to perform interventional procedures within the $\mathrm{O}$-arm fluoroscope. A multi-application electromagnetic surgical navigation system (InstaTrak 3500 Plus ${ }^{\circledR}$, GE Healthcare, Salt Lake City, UT) was used for safe insertion of the laser trocar in percutaneous laser discectomy [2]. Another computer-assisted spinal navigation system (StealthStation ${ }^{\circledR}$, Louisville, CO) was used for screw placement utilizing a percutaneous dynamic reference frame attached to the posterior superior iliac spine for spinal fusion [3].

Although other new and improved computer-assisted spinal navigation systems are now available, they are very expensive and require a large space for set up. PELD requires a variety of systems, including an endoscopic system, fluoroscope, radiofrequency system for ablation or coagulation or laser, basic monitoring system, anesthetic machine, irrigation water system, suction system, light source, and video system. Operating rooms are usually not large enough to accommodate all of these instruments.

In addition, an expert can perform the entire PELD procedure in less than 30 minutes using the conventional approach. There is little need for using these types of heavy, large electromagnetic surgical navigation systems only for increasing the accuracy of the approach with spending over 30 minutes.

A new experimental stereotactic guidance system was designed to facilitate and reduce the time required to determine the needle entry point for discography in uniplanar fluoroscopy-guided PELD. This study was performed to evaluate the feasibility of an experimentally designed stereotactic guidance system for determining needle entry point during uniplanar fluoroscopy-guided intervention.

\section{MATERIALS AND METHODS}

\section{Materials}

1) A conventional method for determining needle entry: Each type of herniated nucleus pulposus (HNP) has a zone and level. First, it is important to identity where the herniation, bulging, protrusion, or extrusion is located in the central zone, subarticular zone, foraminal zone, or extraforaminal zone. Second, each type of HNP may or may not be associated with upward or downward migration [4].

There are 3 types of coronal (frontal), sagittal, and transverse (axial) planes with 3 types of sagittal, transverse (horizontal), and longitudinal (vertical) axes. The needle entry point is determined by the distance from the mid-sagittal plane (transverse axis: $\mathrm{X}$ ). The angle of needle insertion from the skin against the coronal plane (sagittal axis: Y) and against the transverse plane (longitudinal axis: Z) on the lateral view is based on preoperative magnetic resonance imaging (MRI).

A picture archiving and communication system (PACS) drawing tool was used for preoperative determination of the angle and distance from the midline to the skin for the needle entry on an axial film of MRI. The first line was drawn from the skin of the midline via the spinous process to the posterior $1 / 3$ of the intervertebral disc (IVD), and the second line was drawn from the posterior $1 / 3$ of the IVD passing through the herniated disc to the skin. Hence, the distance between the first and second line on the skin was placed on the mid-sagittal plane (transverse axis: $\mathrm{X}$ ). The 2 lines at the posterior 1/3 of the IVD became the angle of needle insertion from the skin against the coronal plane (sagittal axis: Y). The degree of upward or downward migration of herniation affected the needle entry point from above or below the disc level, respectively. It would be the angle of the needle insertion from the skin against the transverse plane (longitudinal axis: Z) (Fig. 1A).

It is essential to adjust the anteroposterior fluoroscopic view in order to clearly visualize both the upper and lower endplate of the targeted disc. According to the conventional method for determining needle entry under fluoroscope, a $\mathrm{K}$-wire is placed between the upper and lower endplate and a line is drawn over the $\mathrm{K}$-wire. A midline is marked between the adjacent spinous processes under fluoroscope. The distance from the midline, which has been measured from preoperative MRI, is marked using a ruler. It is important adjust the lateral view in order to 
clearly visualize both the upper and lower endplates of the targeted disc. It is better to first place a needle to either touch the upper endplate for upward migration or touch the lower endplate for downward migration. Therefore, another $\mathrm{K}$-wire is placed on the lateral view depending on upward or downward migration. The meeting point of the $2 \mathrm{~K}$-wires is the point of needle entry. The angle of needle entry has already been determined from preoperative MRI (Fig. 1B).
2) Design of the stereotactic instrument: A stereotactic system was designed for conveniently for determining needle entry by Kyung-Hoon Kim and it was manufactured by Department of Biomedical Engineering. A 15-cm-long transparent ruler ( $\mathrm{x}$ axis: coronal plane) attaching a wire below its bottom with a $90^{\circ}$ protractor moving from 5 to $15 \mathrm{~cm}$ (angle to coronal plane) has a perpendicular movable wing that has a wire (angle within sagittal plane). The basic
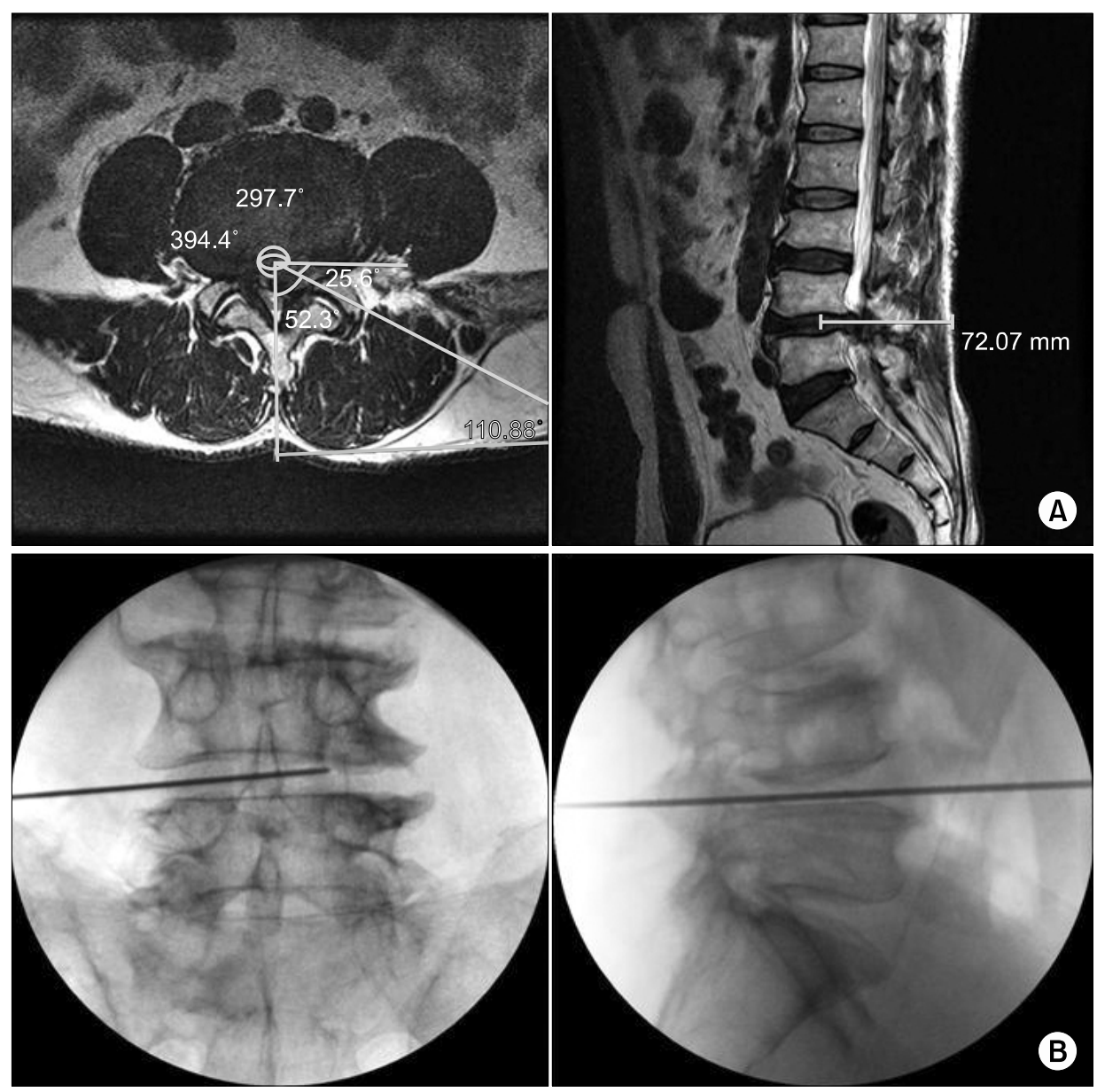

Fig. 1. A conventional method for determining of needle entry on preoperative magnetic resonance image and under fluoroscopy. (A) (Left) Using picture archiving and communication system drawing tool, an angle and the distance from the midline to the skin of the needle entry point are determined preoperatively over an axial film of MRI. The first line is drawn from the skin of the midline via the spinous process to the posterior $1 / 3$ of the intervertebral disc (IVD), and the second line is drawn from the posterior 1/3 of the IVD passing through the herniated disc to the skin. (Right) $A$ line is drawn from the skin to the posterior IVD via the herniated nucleus pulposus on the sagittal view. (B) A conventional method for determining needle entry: (Left) A K-wire is placed between the upper and lower endplate and a line is drawn over the K-wire. The midline is marked between the adjacent spinous processes under fluoroscope. The distance from the midline, which has already been measured from preoperative MRI, is marked using a ruler. (Right) It is important to adjust the lateral view so that both the upper and lower endplate of the targeted disc are clearly seen. It is better to first place a needle to touch the upper endplate for upward migration or to touch the lower endplate for downward migration. According to up- or down-migration, another K-wire is placed on the lateral view. The meeting point of the $2 \mathrm{~K}$-wires is the destination of needle entry. 
stereotactic system was made of acryl, wire, and screws.

However, it is necessary to reduce its weight and volume for sterilization or transport. The stereotactic system was finally completed with following modifications: (1) the heavy non-flexible protractor was removed so that the system could be carried, (2) a tiny roller, instead of a screw, was applied on the middle and both ends to adjust the insertion angles of the needle, and (3) a case for the body, $2 \mathrm{~K}$-wires, and protractor was added so that the system could be carried and sterilized (Fig. 2A).

\section{Methods}

The stereotactic system was used in the clinic to confirm its feasibility. The protocol was approved by the Policy of the Ethical Committee at our Hospital Institutional Review Board. Sixty who received single-level PELD at L4-L5 were randomly divided into the conventional group (Fig. 1B) or the stereotactic group (Fig. 2B). The accuracy of needle entry was evaluated by comparing the distance and angles on postoperative CT with those on preoperative MRI in both groups (Fig. 3).

The duration and radiation exposure for the physician and patient associated with determining the entry point were also compared between both groups. A predicted radiation dose was calculated from electronic dosimeter measurement (Doseguard S $10^{\circledR}$, Raytest, Straubenhardt, Germany) on the infraclavicular fossa of the physician and on the rhombus of Michaelis and the lateral gluteal region

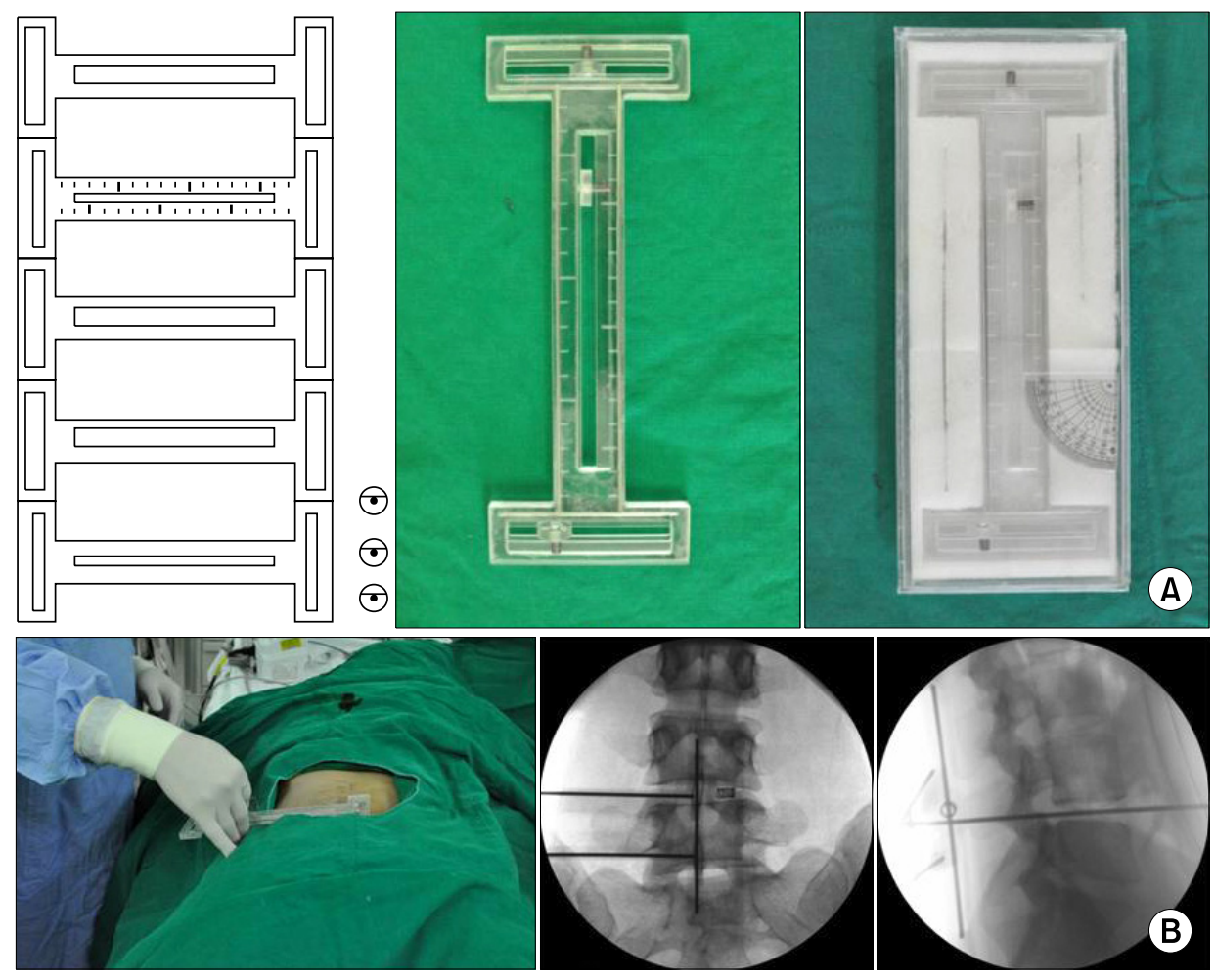

Fig. 2. A stereotactic system for needle entry for percutaneous endoscopic lumbar discectomy. (A) (Left) A computer-aided design was created with a scale for mimicking a stereotactic system. (Middle) A stereotactic system was designed without a scale. A $15 \mathrm{~cm}$-long transparent ruler ( $\mathrm{x}$ axis: coronal plane) attaching a wire below its bottom with a $90^{\circ}$ protractor movable from 5 to $15 \mathrm{~cm}$ (angle to coronal plane) has a perpendicular movable wing which has also a wire (angle within sagittal plane). (Right) A case for the body, 2 K-wires, and protractor allowed the system to be easily carried and sterilized. The stereotactic system was made of acryl, wire, and screws. A tiny roller was applied on the middle and both ends to adjust the insertion angles of the needle. (B) The stereotactic system was applied to patients that underwent a single-level percutaneous endoscopic lumbar discectomy at L4-L5. (Left) The system was placed on the patient to confirm the midline of the spine and the intervertebral space from the anteroposterior view (Middle) and the intervertebral space from the lateral view (Right). A protractor was subsequently placed on the anticipated skin entry point, normally from 8 to $12 \mathrm{~cm}$, according to preoperative magnetic resonance image. 

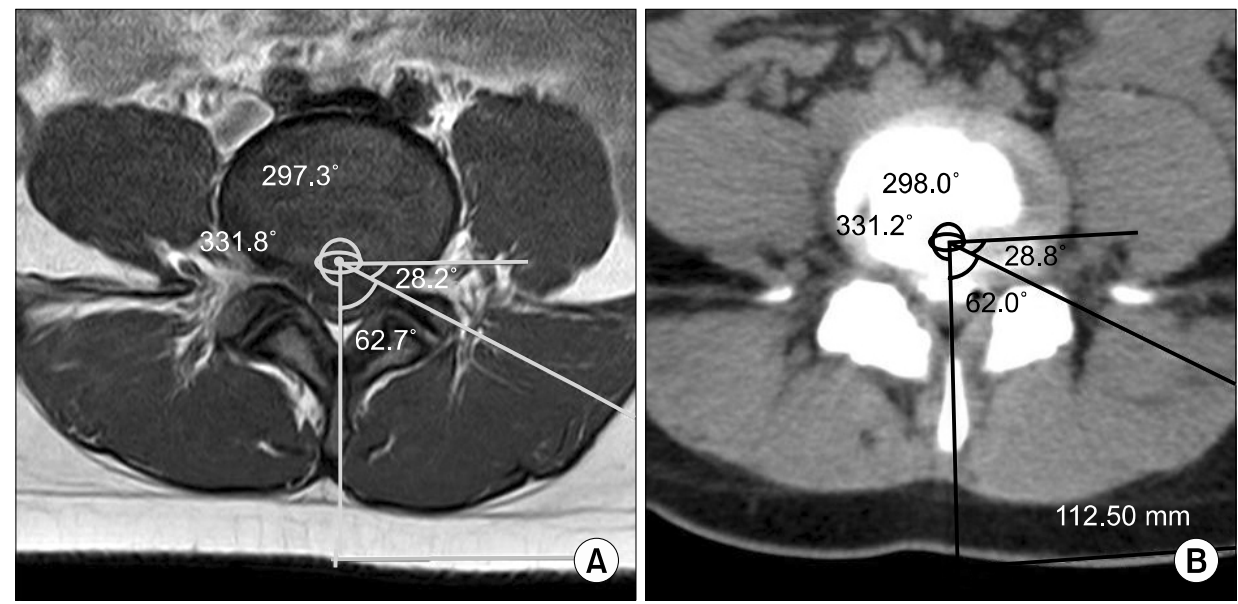

Fig. 3. The accuracy of needle entry by the guidance system was evaluated by comparing the distance and angles between preoperative magnetic resonance image (MRI) and the trace of postoperative computed tomography (CT). (A) Preoperative MRI, (B) Postoperative $\mathrm{CT}$.

Table 1. Mean Distance from the Midline and Mean Angle From the Skin for the Needle Entry Point

\begin{tabular}{|c|c|c|c|c|c|c|}
\hline \multirow{2}{*}{ Methods } & \multicolumn{2}{|c|}{ Preoperative MRI } & \multicolumn{2}{|c|}{ Postoperative CT } & \multirow{2}{*}{$\begin{array}{c}\text { Distance } \\
\text { difference }(\%)\end{array}$} & \multirow{2}{*}{$\begin{array}{c}\text { Angle } \\
\text { difference (\%) }\end{array}$} \\
\hline & Distance (mm) & Angle $\left({ }^{\circ}\right)$ & Distance (mm) & Angle $\left({ }^{0}\right)$ & & \\
\hline Conventional method & $107.40 \pm 2.17$ & $28.67 \pm 1.76$ & $108.39 \pm 2.12$ & $28.16 \pm 30.10$ & $1.25 \pm 0.79$ & $2.92 \pm 1.65$ \\
\hline Stereotactic method & $107.79 \pm 2.13$ & $28.48 \pm 1.48$ & $108.09 \pm 1.77$ & $28.34 \pm 1.36$ & $0.72 \pm 0.45$ & $1.19 \pm 0.79$ \\
\hline
\end{tabular}

Distance and angle difference (\%): the mean absolute value of the percentile change from preoperative to postoperative distance from the midline and angle from the skin.

of the patient.

\section{Statistical analysis}

Statistical analyses were performed using the SPSS program (ver. 12.0 for Windows, SPSS Inc., Chicago, IL). The mean absolute value of the percentile change from the preoperative to postoperative distance from the midline and angle from the skin in both groups were compared using the paired $t$-test with Bonferroni correction. The duration and radiation exposure associated with determining the entry point were compared between the conventional and stereotactic groups by using the Student's t-test with Bonferroni correction. All data are expressed as mean \pm SD. Differences were considered statistically significant when the $P$ value was less than 0.05 .

\section{RESULTS}

From a total of 60 patients, 30 patients randomly assigned into each group and they were measured the distance and angle on the both preoperative MRI and postoperative CT. Significant differences were not observed in the distance $(1.25 \pm 0.79 \mathrm{~mm}$ and $0.72 \pm 0.45 \mathrm{~mm}$; in the conventional and stereotactic groups, respectively) from the midline and angle $\left(2.92^{\circ} \pm 1.65^{\circ}\right.$ and $1.19^{\circ} \pm$ $0.79^{\circ}$; in the conventional and stereotactic groups, respectively) from the skin between preoperative MRI and postoperative CT in both groups (Table 1).

The length of time for determining the entry point under fluoroscope was shortened using the new stereotactic guidance system $(64.3 \pm 5.2 \mathrm{~s})$ compared to the conventional method (113.2 $\pm 8.0 \mathrm{~s})(P<0.05)$. The radiation doses measured in both physician and patient (on the infraclavicular fossa of the physician and on the rhombus of Michaelis and the lateral gluteal region of the patient) were also decreased from $20.0 \pm 2.9,50.6 \pm 8.3$, and 124.0 $\pm 14.6 \mu \mathrm{Sv}$ with the conventional method to $11.9 \pm 1.4$, $27.8 \pm 2.7$, and $58.6 \pm 3.8 \mu \mathrm{Sv}$ with the new stereotactic guidance system, respectively $(P<0.05)$ (Table 2$)$.

No complications were observed with the new stereotactic system or the conventional method.

\section{DISCUSSION}

The new stereotactic guidance system saved time and provided reliable guidance to for determining the needle 
Table 2. The Durationand Radiation Exposure for Determining the Entry Point Under Fluoroscope

\begin{tabular}{|c|c|c|c|c|}
\hline \multirow{3}{*}{ Methods } & \multirow{3}{*}{ Time (s) } & \multicolumn{3}{|c|}{ Radiation exposure ( $\mu \mathrm{S} v)$} \\
\hline & & \multirow{2}{*}{ Physician } & \multicolumn{2}{|c|}{ Patient } \\
\hline & & & $\mathrm{AP}$ & Lateral \\
\hline Conventional method & $113.2 \pm 8.0$ & $20.0 \pm 2.9$ & $50.6 \pm 8.3$ & $124.0 \pm 14.6$ \\
\hline Stereotactic method & $64.3 \pm 5.2^{*}$ & $11.9 \pm 1.4^{\dagger}$ & $27.8 \pm 2.7^{\ddagger}$ & $58.6 \pm 3.8^{\S}$ \\
\hline
\end{tabular}

Physician: A predicted radiation dose calculated from dosimeter measurement when the dosimeter was placed on the infraclavicular fossa of the physician. AP: A predicted radiation dose calculated from dosimeter measurement when the dosimeter was placed on the rhombus of Michaelis of the patient. Lateral: A predicted radiation dose calculated from dosimeter measurement when the dosimeter was placed on the lateral gluteal region of the patient. ${ }^{*, \uparrow, \uparrow, \delta}$ Duration and radiation exposure were reduced in the stereotactic method group compared with the conventional method group.

entry point for discography and consecutive PELD. However, the stereotactic system version 2 requires improvements such as lighter weight and increased reproducibility.

An ideal stereotactic system requires accurate guidance to the target. Factors, such as patient movement during the operation, change of lumbar lordosis in the prone position with different pillow heights, or change in the angle of the fluoroscopic view, may result in a different distance or angle of needle entry.

Previous reports have stated that compared to open surgery, minimally invasive spine surgery (MISS) has great merits, including lower infection rates, more tolerate postoperative pain, and a shorter hospital stay. A drawback of this approach is that multiple fluoroscopic images must be obtained to ensure correct tube positioning; this exposes the surgeon and surgical team to increased doses of ionizing radiation relative to those in traditional open procedures. Surgeons performing minimally invasive operations receive 10 to 22 times more radiation than that received by surgeons performing open operations [5].

PELD is a mainstay of MISS. Sufficient analgesia during the surgery makes the patient feel as if it is real MISS. The preferred route of performing PELD is under local anesthesia with intravenous analgesia rather than under general anesthesia, which is accompanied by complicated neural monitoring systems and risks such as accidental extubation or disposition of the endotracheal tube during surgery with the patient in a prone position [6]. Monitored anesthetic care greatly facilitates to the performance of MISS. Dexmedetomidine ensures cooperative sedation during the operation without the risk of respiratory depression [7]. Dexmedetomidine is administrated at least 10 minutes before surgery and is titrated intraoperatively to ensure that the patient will be appropriately sedated and will not move during surgery. In addition, a preoperative analgesic patch of $5 \%$ lidocaine provides pain relief during PELD, especially at the stages of needle insertion, skin incision, serial dilation and insertion of the working channel, and subcutaneous suture [6]. During surgery, anular infiltration of $1 \%$ lidocaine helps to reduce pain-related motion of the patient unconsciously. Traditionally, intravenous analgesia with local skin infiltration has been used to prevent $\mathrm{pa}-$ tient's movement resulting from incisional pain.

To accurately target the HNP, it is important to properly place the needle for discography and subsequent discectomy. A "push and pull technique" is the best and quickest method for a contained HNP based on preoperative MRI evaluation [8]. "Pushing the HNP into the anterior $1 / 3$ of the disc and then pulling it out" is a simple technique under fluoroscopy with/without endoscopic vigilance. However, both the distance from the midline and the angle from the skin may result in needle entering at a different point based on the direction of herniation in downward or upward migration or to the central, subarticular, foraminal, or extraforaminal zone [4].

Many manufactures have produced different types of surgical navigation systems for spine surgery. The main purpose of these systems is to provide an accurate insertion point for the best route to the target organ with minimal destruction of normal tissues. However, the main concerns of using these systems are radiation exposure from using an image-guided navigation system and costeffectiveness. Most operating rooms do not have adequate space for accommodating these surgical navigation systems.

Thus far, clinical data strongly supporting the use of image-guidance techniques have only been published for pedicle screw implantation in the cervical and lumbar spine [9]. A major advantage of image-guidance system-based 
education modules, if systematically used in a lab setting, is that they allow a trial-and-error based educational approach. Interesting developments are expected from the integration of image analysis techniques and endoscopy. Image-guided techniques have reached a high level of development, as the accuracies that can be achieved technically meet the anatomical demands [10]. Intraoperative CT or MRI is also now available in the surgical field [11-13]. The utility of robotics or 3-dimensional CT is determined by cost and time benefits and outcomes of research on both safety and efficacy issues [14,15].

Intraoperative, full-rotation, 3-dimensional image (O-arm) fluoroscopy may help navigation to the targeted organ in spine surgeries or interventions with reduced radiation exposure [16,17]. In addition, compared with the use of traditional systems of image acquisition and registration for navigation, the use of the commercially available navigation system with the $\mathrm{O}$-arm system showed a higher accuracy for spinal navigation [18]. However, during the operation, surgeons are required to operate inside the machine within a limited space.

The great merits of this stereotactic system for PELD include its low cost, easy adaptability for users who are accustomed to the conventional method, and the fact that it does not occupy a large space. For the confirmation of a safe unipedicular vertebroplasty, an imaginary approach line is drawn on the skin using preoperative CT or MRI and a PACS drawing tool [19]; therefore, this system may be helpful during the use of the unipedicular approach for vertebroplasty, which has a risk of neural damage. However, this system could be improved by smoothing the rotation movement of the $\mathrm{K}$-wires, reducing the weight, and increasing the flexibility.

The new stereotactic guidance system under fluoroscopy is feasible and time-saving experimental design for determining needle entry point during uniplanar fluoroscopy-guided intervention. Therefore, this system may reduce radiation exposure-, which is the main drawback of MISS. However, several limitations of this design, such as heaviness, difficulty in rotation movement, and lack of flexibility, should be overcome.

\section{ACKNOWLEDGEMENTS}

This study was supported by 2-year Pusan National University Research Grant.

\section{REFERENCES}

1. Park MS, Lee KM, Lee B, Min E, Kim Y, Jeon S, et al. Comparison of operator radiation exposure between $\mathrm{C}$-arm and $\mathrm{O}-$ arm fluoroscopy for orthopaedic surgery. Radiat Prot Dosimetry 2012; 148: 431-8.

2. von Jako RA, Cselik Z. Percutaneous laser discectomy guided with stereotactic computer-assisted surgical navigation. Lasers Surg Med 2009; 41: 42-51.

3. Best NM, Sasso RC, Garrido BJ. Computer-assisted spinal navigation using a percutaneous dynamic reference frame for posterior fusions of the lumbar spine. Am $J$ Orthop (Belle Mead NJ) 2009; 38: 387-91.

4. Kim KH, Kim IS. Current understanding of spinal pain and the nomenclature of lumbar disc pathology. In: Minimally invasive percutaneous spinal techniques. Edited by Kim DH, Kim KH, Kim YC. Philadelphia, Elsevier/Saunders. 2010, p 44.

5. Ropper AE, Chi JH. Maximal radiation exposure during minimally invasive spine surgery? Neurosurgery 2011; 68: N23-4.

6. Kim KH. Use of lidocaine patch for percutaneous endoscopic lumbar discectomy. Korean J Pain 2011; 24: 74-80.

7. Harned ME, Owen RD, Steyn PG, Hatton KW. Novel use of intraoperative dexmedetomidine infusion for sedation during spinal cord stimulator lead placement via surgical laminectomy. Pain Physician 2010; 13: 19-22.

8. Yeung AT. The evolution of percutaneous spinal endoscopy and discectomy: state of the art. Mt Sinai J Med 2000; 67: 327-32.

9. Tian NF, Huang QS, Zhou P, Zhou Y, Wu RK, Lou Y, et al. Pedicle screw insertion accuracy with different assisted methods: a systematic review and meta-analysis of comparative studies. Eur Spine J 2011; 20: 846-59.

10. Tjardes T, Shafizadeh S, Rixen D, Paffrath T, Bouillon B, Steinhausen ES, et al. Image-guided spine surgery: state of the art and future directions. Eur Spine J 2010; 19: 25-45.

11. Tonn JC, Schichor C, Schnell O, Zausinger S, Uhl E, Morhard D, et al. Intraoperative computed tomography. Acta Neurochir Suppl 2011; 109: 163-7.

12. Schlenzka D, Laine T, Lund T. Computer-assisted spine surgery. Eur Spine J 2000; 9 Suppl 1: S57-64.

13. Jolesz FA. Future perspectives for intraoperative MRI. Neurosurg Clin N Am 2005; 16: 201-13.

14. Specht LM, Koval KJ. Robotics and computer-assisted orthopaedic surgery. Bull Hosp Jt Dis 2001-2002; 60: 168-72.

15. Barzilay $Y$, Liebergall M, Fridlander A, Knoller N. Miniature robotic guidance for spine surgery--introduction of a novel system and analysis of challenges encountered during the clinical development phase at two spine centres. Int J Med Robot 2006; 2: 146-53.

16. Ishikawa Y, Kanemura T, Yoshida G, Matsumoto A, Ito Z, 
88 || Korean J Pain Vol. 25, No. 2, 2012

Tauchi R, et al. Intraoperative, full-rotation, three-dimensional image (0-arm)-based navigation system for cervical pedicle screw insertion. J Neurosurg Spine 2011; 15: 472-8.

17. Abul-Kasim K, Söderberg M, Selariu E, Gunnarsson M, Kherad M, Ohlin A. Optimization of radiation exposure and image quality of the cone-beam O-arm intraoperative imaging system in spinal surgery. J Spinal Disord Tech 2012; 25: $52-8$.
18. Oertel MF, Hobart J, Stein M, Schreiber V, Scharbrodt W. Clinical and methodological precision of spinal navigation assisted by 3D intraoperative 0 -arm radiographic imaging. J Neurosurg Spine 2011; 14: 532-6.

19. Kim KH, Kim TK. Unipedicular approach for percutaneous vertebroptasty. Edited by Kim DH, Kim KH, Kim YC. Philadelphia, Elsevier/Saunders. 2010, pp 270-6. 